\title{
LDscaff: LD-based scaffolding of de novo genome assemblies
}

\author{
Zicheng Zhao ${ }^{1,2+}$, Yingxiao Zhou ${ }^{1,4+}$, Shuai Wang ${ }^{2}$, Xiuqing Zhang ${ }^{1}$, Changfa Wang ${ }^{3^{*}}$ and Shuaicheng Li $^{2^{*}}$
}

From The International Conference on Intelligent Biology and Medicine (ICIBM) 2020 Virtual. 9-10 August 2020

\author{
*Correspondence: \\ wangcf1967@163.com; \\ shuaicli@cityu.edu.hk \\ 'Zicheng Zhao and Yingxiao \\ Zhou have contributed \\ equally to this study. \\ 2 Department of Computer \\ Science, City University \\ of Hong Kong, Kowloon, \\ Hong Kong SAR 999077, \\ China $^{3}$ Liaocheng Research \\ Institute of Donkey \\ High-Efficiency Breeding \\ and Ecological Feeding, \\ Liaocheng University, \\ Liaocheng City 252059, \\ Shandong, China \\ Full list of author information \\ is available at the end of the \\ article
}

\begin{abstract}
Background: Genome assembly is fundamental for de novo genome analysis. Hybrid assembly, utilizing various sequencing technologies increases both contiguity and accuracy. While such approaches require extra costly sequencing efforts, the information provided millions of existed whole-genome sequencing data have not been fully utilized to resolve the task of scaffolding. Genetic recombination patterns in population data indicate non-random association among alleles at different loci, can provide physical distance signals to guide scaffolding.

Results: In this paper, we propose LDscaff for draft genome assembly incorporating linkage disequilibrium information in population data. We evaluated the performance of our method with both simulated data and real data. We simulated scaffolds by splitting the pig reference genome and reassembled them. Gaps between scaffolds were introduced ranging from 0 to $100 \mathrm{~KB}$. The genome misassembly rate is $2.43 \%$ when there is no gap. Then we implemented our method to refine the Giant Panda genome and the donkey genome, which are purely assembled by NGS data. After LDscaff treatment, the resulting Panda assembly has scaffold N50 of $3.6 \mathrm{MB}, 2.5$ times larger than the original N50 (1.3 MB). The re-assembled donkey assembly has an improved N50 length of $32.1 \mathrm{MB}$ from $23.8 \mathrm{MB}$.
\end{abstract}

Conclusions: Our method effectively improves the assemblies with existed resequencing data, and is an potential alternative to the existing assemblers required for the collection of new data.

Keywords: De novo assembly, Maximum weighted matching, Linkage disequilibrium

\section{Background}

With the massive increases in the throughput of the Next Generation Sequencing (NGS) technique, a large number of organisms have been sequenced and assembled [1-8]. Most current assembly approaches stitched short reads together to generate contigs and scaffolds. Though NGS provides accurate base-level sequences, specific regions such as nonrandom repeat elements can hardly be accurately assembled. The reason for the author(s) and the source, provide a link to the Creative Commons licence, and indicate if changes were made. The images or other third party material in this article are included in the article's Creative Commons licence, unless indicated otherwise in a credit line to the material. If material is not included in the article's Creative Commons licence and your intended use is not permitted by statutory regulation or exceeds the permitted use, you will need to obtain permission directly from the copyright holder. To view a copy of this licence, visit http:// creativecommons.org/licenses/by/4.0/. The Creative Commons Public Domain Dedication waiver (http://creativecommons.org/publi cdomain/zero/1.0/) applies to the data made available in this article, unless otherwise stated in a credit line to the data. 
contiguity problem is typical short reads with lengths in the range of 20-500 bp can hardly cover the repeat regions [9-11]. Also, vulnerable spots that may introduce gaps in the assembly process $[9,11,12]$ due to uneven sequencing coverage.

Long-range scaffolding technologies can provide long-range connectivity, which can also aid in resolving the complex regions. Such methods include end sequencing of fosmid clones [1], fosmid-based dilution pool sequencing [13, 14], optical mapping [15-17], genetic mapping with restriction site associated DNA (RAD) tags [18] and proximity ligation $(\mathrm{Hi}-\mathrm{C})$ sequencing. However, each of these methods has a limitation in either experimental cost or application scenarios [19]. Fosmid cloning is sensitive to the quantity and quality of the input DNA, while fosmid libraries are subject to cloning bias. The data generating process for optical map construction involves mostly manual steps. These steps include DNA extension and image capture, which are low throughput and inefficient. Genetic maps are costly or impractical to generate from many species. Although the Hi-C data provide extensive links covering large distances, the current resolution is not high enough for the local ordering of small adjacent contigs.

Linkage disequilibrium(LD) is the non-random association of alleles at different loci in population genetics $[20,21]$. LD is of importance in population genetics because it reflects evolutionary history. It is derived from several population genetic forces that structure a genome, such as population selection, recombination, mutation, genetic drift, mating rate, population sex ratio, and genetic linkage. Genetic linkage maps constructed from population data now provide the basis for a wide range of genomics studies. LD depends strongly on one-dimensional distance and can extend over $550 \mathrm{~KB}$ [22]. LODE [23] uses this kind of linkage information to place unpositioned SNPs by estimating LD with SNPs with confirmed locations. LD maps constructed from SNP data can guide the ordering of contigs from a $216 \mathrm{~KB}$ region [24]. Thus, the high-density inter-marker LD in the population dataset has the potential to inform the orders and orientation of scaffolds over a large distance. Some methods have been published to integrate whole genome sequencing(WGS) data and linkage map construction. POPSEQ [25] requires samples from a known crossing design to assemble a barley genome. Recombinant Population Genome Construction [26] first build a 'consensus' assembly from sequencing a population of recombinant individuals, then a linkage map was generated to improve the assembly. The joint assembly and mapping method [27] constructs a high-density genetic map to exam the genome organization. Either these methods require specific crossing designs or a built linkage map. According to our best knowledge, there is no available tool to guide scaffolding based on LD without building linkage maps.

Population analysis is essential in species genome studies to investigate the structural and variants among individuals as well as their evolutionary history. Currently, a large number of draft genomes in NCBI are assembled purely by short sequence reads. Accompanied by these draft genomes, whole-genome resequencing data have also experienced rapid development but have not obtained considerable integration and manipulation. Here we present $L D s c a f f$ to consider whether linkage information obtained from single-nucleotide variations in population, combined with short reads data, is capable of providing extra information in scaffold contiguity. LDscaff aids in the layout of a set of scaffolds with a graph method, by taking as input the population variation data, a set of 
scaffolds, to build an undirected graph with a set of vertices and edges, representing the scaffolds and the LD strength among them. Computing the optimal orders and orientations of these scaffolds can be treated as a maximum weight matching problem.

We applied our method to both simulated data and empirical data to verify the effectiveness of our method. The simulation experiments were performed on a pig genome. We randomly split the pig reference genome [28] into 360 scaffolds and tried to reassemble them. The average error rate (percentage of misassemblies) is $2.43 \%$ in 20 experiment trials. We then refined the draft Giant Panda genome [4] and a de novo donkey genome assembly. These draft assemblies were both assembled using only short reads. The resulting Giant Panda assembly has a scaffold N50 of 3.6 MB, 2.5 folders larger than the original one. The re-assembled donkey assembly has an improved N50 length of 32.1 MB.

\section{Implementation}

\section{The scaffolding problem}

The principle of whole-genome shotgun assemblies is to assign, order, and orient sequence contigs. Our method solves the scaffolding problem with a graphical algorithm. We build a complete graph $G$, with vertices $V$ representing scaffolds, and edge weights $E$ corresponding to the linkage power between pairs of scaffolds. Given a weighted graph $G$, the problem is transformed into finding a set of edges that have the maximum sum-up weight and do not share common vertices. The problem is known as the maximum matching problem in graphical theory.

\section{Data prepossessing}

We downloaded the giant panda reference AilMel 1.0 from the NCBI GenBank database (Accession number: GCA000004335.1). The genome coverage is 60x, and the N50 of contigs and scaffolds are 39,886 bp and 1,281,781 bp respectively. We downloaded the chromosome-level panda genome [29] from the National Genomics Data Center (Accession Number: GWHACDL00000000). The genome coverage is $82 x$, and the genome sequence N50 is $129,245,720 \mathrm{bp}$.

A purebred donkey individual was sequenced on an Illumina HiSeq 2000 sequencing platform. The paired-end reads were initially assembled with SOAPdenovo v2.04.4 [30] to construct short but accurate scaffolds. Tiny scaffolds shorter than $2 \mathrm{~KB}$ (containing $5.45 \%$ sequence bases) were set aside for insufficient linkage signal, and 2974 scaffolds remained. The assembly contained $2.4 \mathrm{~Gb}$ of sequence (scaffold N50: 22.0 MB).

We downloaded the panda population data from the NCBI Short Read Archive (SRA) database under accession SRA053353. In total, we obtained 34 panda samples, and then we aligned them to the reference with the BWA aln algorithm (Version 0.7.13) [31]. The bam files were converted and sorted using Samtools (Version 1.3.1) [32]. We used Freebayes (Version 0.9.10) [33] to call SNPs with default parameters. After that, we used GATK VariantFiltration [34] to extract reliable variants with strict criteria ( $Q D<2.0, F S>60.0, M Q<40.0$, MQRankSum $<12.5$, ReadPosRankSum $<8.0 \quad$ ). Sorted BAM files were recalibrated with GATK BQSR with reliable SNPs. Local realignment around indels was performed with reliable indels using GATK Indel Realigner. 
SNPs were called and filtered using GATK HaplotypeCaller with the same strict criteria as above. In total, we obtained 13 million $(13,427,006)$ SNPs.

Population resequencing data from 132 donkeys were collected. These samples were sequenced on an HiSeq 4000 platform. Trimmomatic [35] was used to remove adaptor contamination. Cleaned reads were mapped to the donkey assembly using BWA (version: 0.7.10-r789). High-quality reads were selected $(M Q>20)$ with SAMTools (version 1.3.1). Regions covered by at least two reads in most samples (80\%) were extracted. Duplications were marked and removed with Picard. High confident SNPs were obtained after GATK HaplotypeCaller and hard filtering were conducted $(Q D<2.0, F S>60.0, M Q<40.0, M Q R a n k S u m<12.5)$. Finally, the original BAM files were recalibrated with GATK BQSR with this set of SNPs. Among final SNPs, variants with small minor allele frequency less than 0.2 were filtered out.

\section{Building scaffolding graph}

Denote the graph $\mathrm{G}$ with the vertex set $\mathrm{V}$ and edge set $\mathrm{E}$, each vertex refers to one side of a scaffold. For $\mathrm{n}$ scaffolds, the number of vertices in $\mathrm{G}$ is $2 \mathrm{n}$. Edges are added for any two vertices. The graph is shown in Fig. 1a. We classify the edges into two groups, inneredges $E_{i}$, and outer-edges $E_{0}$. An inner-edge (blue lines in Fig. 1a) connects two vertices that belong to the same scaffold, while an outer-edge (black lines in Fig. 1a) does not. The weight of an outer-edge is the linkage power between the two corresponding vertices. The inner-edges have weights of zero.

\section{Linkage power calculation}

Each outer-edge connects two distinct vertices, referring to two scaffold ends. For each scaffold end, $m$ markers ( $m=100$ by default) were extracted. Two-loci LD is calculated for $m^{*} m$ marker pairs. Linkage power for two scaffold ends is the average value for all pairwise LD statistics. Short scaffolds (with markers fewer than $\mathrm{m}$ ) were set aside for insufficiency to provide linkage information.

The linkage power between the two sides can be considered as the approximation of the physical distance between the vertices-the larger the linkage power, the shorter
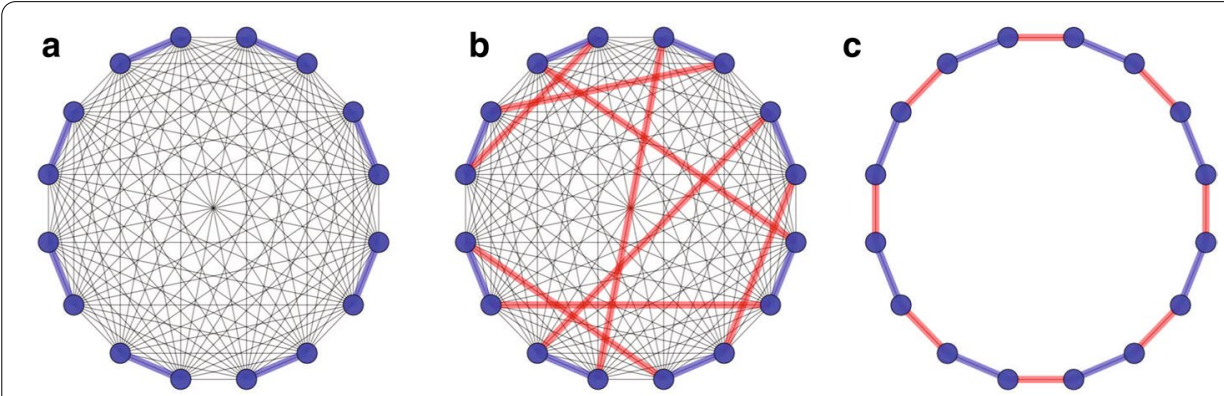

Fig. 1 Illustration of the algorithm. a A complete graph constructed from eight scaffolds. The blue lines are the inner-edges that represent the vertexes belong to the same scaffold. The black lines are the outer-edges that represent the linkage between vertexes that are in the different scaffolds; $\mathbf{b}$ The set of red lines is a solution of the matching problem for the complete graph in $\mathbf{a}$. All the red lines do not share comm-on vertexes; c The solution of the matching problem (red lines) and the inner-edges (blue lines) can form a circle. The linear permutation and the orientation of the scaff-olds can be obtained by remove the red line with the lowest linkage signal 
physical distances on the genome. In reality, the inference of distance proximity from residue pair linkage is susceptible to both false negative and false positives. In our cases, allele distributions are estimated from a finite sample. Thus, a spurious nonzero allele frequency is likely to contribute a large LD value. To solve this issue, we estimated the LD using balanced allele distribution.

Allele frequencies are balanced as follows. Consider two positions having two possible alleles, with alleles $A, a$ at the first site and $B, b$ at second site respectively, there are 9 possible genotype combinations. We use $k_{i}$ to denote the occupancy of each possible genotype. We also define $d_{i}$ as the number of samples to remove. All possible combinations are displayed in Table 1.

Allele frequencies for allele $A$ and for allele $B$ are introduced,

$$
\begin{aligned}
f_{A} & =\frac{2 \sum_{i \in\{1,2,3\}}\left(k_{i}-d_{i}\right)+\sum_{i \in\{4,5,6\}}\left(k_{i}-d_{i}\right)}{2 \sum_{i=1}^{9}\left(k_{i}-d_{i}\right)} \\
f_{B} & =\frac{2 \sum_{i \in\{1,4,7\}}\left(k_{i}-d_{i}\right)+\sum_{i \in\{2,5,8\}}\left(k_{i}-d_{i}\right)}{2 \sum_{i=1}^{9}\left(k_{i}-d_{i}\right)}
\end{aligned}
$$

We expect the allele frequency of allele $A$ and allele $B$ are approximate to 0.5 with the minimum number of removed individuals. We balance the frequencies using integer linear programming.

$$
\min \sum d_{i}
$$

subject to

$$
\begin{aligned}
& \left|f_{A}-0.5\right| \leqslant \theta \\
& \left|f_{B}-0.5\right| \leqslant \theta \\
& d_{i} \geq 0, \forall i
\end{aligned}
$$

The linkage disequilibrium denoted as $D_{p}$ is then calculated by the Fisher's exact test with the balanced allele frequencies.

\section{Permutation and orientation}

The solution for maximum matching in $G$ indicates the orders and orientation of the scaffolds. The maximum matching only includes outer-edges, and the degree of each vertex equals one as shown in Fig. 1 . The matching constructed by $E_{i}$ will form one or several circles in $G$ with all the vertices in them (Fig. 1). Each circle can be transformed into a linear path by removing the outer edge with the weakest weight in $e_{M}$.

\section{Software implementation}

There are several algorithms for solving the maximum matching problem in a general graph [36-38]. In LDscaff we applied Edmond's blossom shrinking algorithm implemented by lemon [39] - an open-source graph library written in the $\mathrm{C}++$ language. 

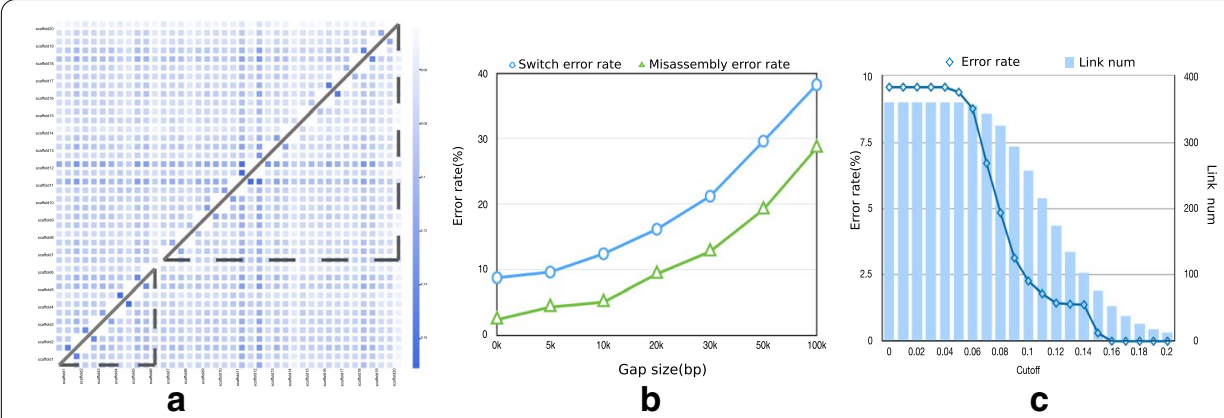

Fig. 2 LDscaff performance in simulated data. a The heatmap for the LD ma-trix in the simulated experiment with 40 vertices in 20 scaffolds, pixel intensity in the matrix indicates the strength of LD. Solid lines represent correct scaffolding orders, while dash lines represent misassembly; b The error rates versus gap sizes; c The error rates and numbers of matching links using different cutoff thresholds

Table 1 Enumeration of the genotypes and the notations

\begin{tabular}{llll}
\hline Locus 1 & Locus 2 & Observed individual number & $\begin{array}{l}\text { Withdraw } \\
\text { individual } \\
\text { number }\end{array}$ \\
\hline $\mathrm{AA}$ & & $k_{1}$ & $d_{1}$ \\
$\mathrm{AA}$ & $\mathrm{BB}$ & $k_{2}$ & $d_{2}$ \\
$\mathrm{AA}$ & $\mathrm{Bb}$ & $k_{3}$ & $d_{3}$ \\
$\mathrm{Aa}$ & $\mathrm{bb}$ & $k_{4}$ & $d_{4}$ \\
$\mathrm{Aa}$ & $\mathrm{BB}$ & $k_{5}$ & $d_{5}$ \\
$\mathrm{Aa}$ & $\mathrm{Bb}$ & $k_{6}$ & $d_{6}$ \\
$\mathrm{aa}$ & $\mathrm{bb}$ & $k_{7}$ & $d_{7}$ \\
$\mathrm{aa}$ & $\mathrm{BB}$ & $k_{8}$ & $d_{8}$ \\
$\mathrm{aa}$ & $\mathrm{Bb}$ & $k_{9}$ & $d_{9}$ \\
\hline
\end{tabular}

\section{Evaluating assemblies}

To evaluate the quality of assemblies, we mapped all assemblies to the corresponding reference genomes with nucmer [40] using the default parameters. We used QUAST [41] to collect various metrics (command line: “-eukaryote -min-contig 3000 -min-alignment 500 -extensive-mis-size 7000 -fast -split-scaffolds").

\section{Results}

We performed experiments on both simulated data and empirical data to evaluate the accuracy and effectiveness of our method.

\section{Simulation result}

We simulated scaffolds by randomly splitting the pig assembly Sus scrofa 10.2 and applied LDscaff on the generated scaffolds to check whether it can produce the correct orders and orientations. For better illustration, we first select chr1 in Sus scrofa 10.2, and simulated input scaffolds by splitting the chromosome into 20 scaffolds. We created a complete graph with 40 vertices as demonstrated in the "Method" section. Linkage strength between any scaffold ends is calculated using 100 SNPs. The heatmap in Fig. 2a shows the corresponding LD matrix of scaffold end pairs. The vertices were labeled by 
Table 2 Comparison of the refined panda genome with SOAPdenovo-assembled genome

\begin{tabular}{lllll}
\hline Cutoff & Scaffolds before & Scaffolds after & N50 before & N50 after \\
\hline 0.1 & 57 & 57 & 1335486 & 1158329675 \\
0.15 & 57 & 254 & 1335486 & 20717847 \\
0.2 & 57 & 1344 & 1335486 & 3358889 \\
0.25 & 57 & 2123 & 1335486 & 1962870 \\
0.3 & 57 & 2509 & 1335486 & 1625199 \\
0.4 & 57 & 2843 & 1335486 & 1413235 \\
0.5 & 57 & 2953 & 1335486 & 1353331 \\
0.6 & 57 & 2981 & 1335486 & 1336590 \\
0.7 & 57 & 2983 & 1335486 & 1335486 \\
\hline
\end{tabular}

the scaffold position order in reference. Orders of the vertices represent a permutation of the original scaffold ordering. Pixel intensity in the matrix indicates the strength of LD.

There are two patterns as showed in the heatmap. The first pattern is that the LD value between scaffold end pairs decay as their genomic distance increases. Adjacent vertices tend to have higher weights of LD. The second is that scaffolds from different chromosomes can be clustered utilizing the boundaries of the heatmap.

In Fig. 2a, solid lines that link diagonal white boxes imply correct scaffolding orders. One misassembly was outlined with two sets of dashed lines, one links scaffold1 and scaffold6 together, the other one links scaffold7 and scaffold20 together. We denoted the error rate as the percentage of misjoins of all scaffolds, evaluating the accuracy of LDscaff. The error rate, in this case, is $1 / 40=2.5 \%$. We also extended the experiments to the whole genome-wide, we split the 18 chromosomes in the of Sus scrofa 10.2 by randomly splitting the 18 chromosomes into 360 scaffolds. We then built a graph and resolve the layout of the simulated scaffolds. With 20 experimental repeats, the average error rate is $2.43 \%$.

As the LD linkage disequilibrium decays with the increase of the physical distance of the loci in the genome, we test how the performance of scaffolding is affected by gap sizes between the simulated scaffolds. We introduce gaps ranging from 5 to $100 \mathrm{~KB}$ respectively. To estimate the accuracy better, we introduced the switch error rate, which is the number of switches that required for transforming the solution into the correct matching divided by the total number of links. The error rates and the switch error rates for different gap sizes are shown in Fig. 2b. As expected, both kinds of error rates increase as the gap size increases. When the gap size becomes larger, false-positive signals tend to increase. Different cutoff thresholds to break to weakest links were also tested as shown in Fig. 2c.

\section{The giant panda genome}

Based on the previously published panda assembly AilMel 1.0 purely from short reads, and a chromosome-level reference genome assembly (GWHACDL00000000) using linked-reads, we were able to test the accuracy of scaffolding. The AilMel 1.0 assembly was refined with LDscaff. After filtering, 2983 contigs remained and were linked into sets of cycles using different cutoff thresholds, as shown in Table 2. We then compared the 
generated sets of giant panda scaffolds with the GWHACDL00000000 assembly using mummer 3.23 [42] as shown in Fig. 2a, b using cutoff of 0.1 and 0.2, respectively. The complete alignment results with cutoffs $0.1,0.2$, and 0.3 are shown in Additional file 1: Figs. S1-S22. We broke the links that have LD weight less than 0.2 (termed as the cutoff), we get improved N50 (3.6 MB) 2.5 folder larger than the original one (1.3 MB) as shown in Table 2. Qualities of AilMel 1.0 assembly and refined assembly were evaluated using QUAST, the metric was shown in Table 3.

\section{The donkey genome}

We applied our approach to improving a donkey genome assembly. We calculated scaffold pairwise LD strength with 100 SNPs at each side of the scaffold, scaffolds that not long enough to provide sufficient SNPs were filtered out. We then link the remaining 551 scaffolds using linkage information. The raw resulting assembly consisted of 80 cycles, they were then partitioned into 382 scaffolds (N50 length of $32.1 \mathrm{MB}$ ) after breaking the links with a cutoff of 0.2 . The assembly now contains $94.3 \%$ of the total sequence (2.27G). The relationship of different cutoff thresholds and N50 of the refined assembly is shown in Table 4. The misassembly evaluation comparison metric of draft assembly and the refined assembly (cutoff equals 0.2 ) is shown in Table 5 .

To investigate the refinement of the donkey genome in detail, we performed synteny comparison to the horse genome as shown in Fig. 3c, $d$ using cutoffs of 0.1 and 0.2. The full alignment results were shown in Additional file 1: Figs. S23-S54. We first sorted donkey scaffolds and oriented them according to horse chromosomes (hereafter, labeled as ECA). Consider the absence of genomes from closest phylogenetic relatives(rhinos), we only take rearrangements occurring within donkey scaffolds as reliable. More strictly, only rearrangements that pass all cutoff thresholds are further investigated. We identified two inversions between the horse and the donkey, that mapped to ECA7 and ECA28, as shown in Fig. 4. Both of them have been verified in a de novo donkey genome by Chicago HiRise assembly [43]. Around regions these found inversions, LD was ranked top in genome-wide level, which is consistent with the algorithm that LDscaff used. Setting high cutoff can distinguish reliable inversions from false-positive ones caused by misassembly. It implies that $L D$ scaff has the potential to aid in the identification of chromosome inversions.

\section{Comparison of $\mathrm{HiC}$ and LDscaff}

We used $\mathrm{HiC}$ data to reassemble the same draft donkey genome from SOAPdenovo2, and the re-assembled scaffolds using LDscaff. The assembly was scaffolded with Hi-C data using the 3D-DNA pipeline [44]. The Hi-C reads were aligned to the draft donkey genome assembly using the Juicer pipeline [45]. The 3D-DNA pipeline was run with the default parameters. We evaluated assembly across four categories of error: relocations, translocation, inversion, and indels. The full comparison results are shown in Table 6. The number and size of these errors were calculated after QUAST splits input assembly by continuous fragments. For assembles involved $\mathrm{HiC}$ scaffolding, contigs shorter than $15 \mathrm{~KB}$ were set aside. Compared with $\mathrm{HiC}$ scaffolding, LDscaff-reassembled sequence has less scaffolding errors decreased with decreasing assembly contiguity. The His-reassembled sequence has twice misassembled bases more than the LDscaff result. When 

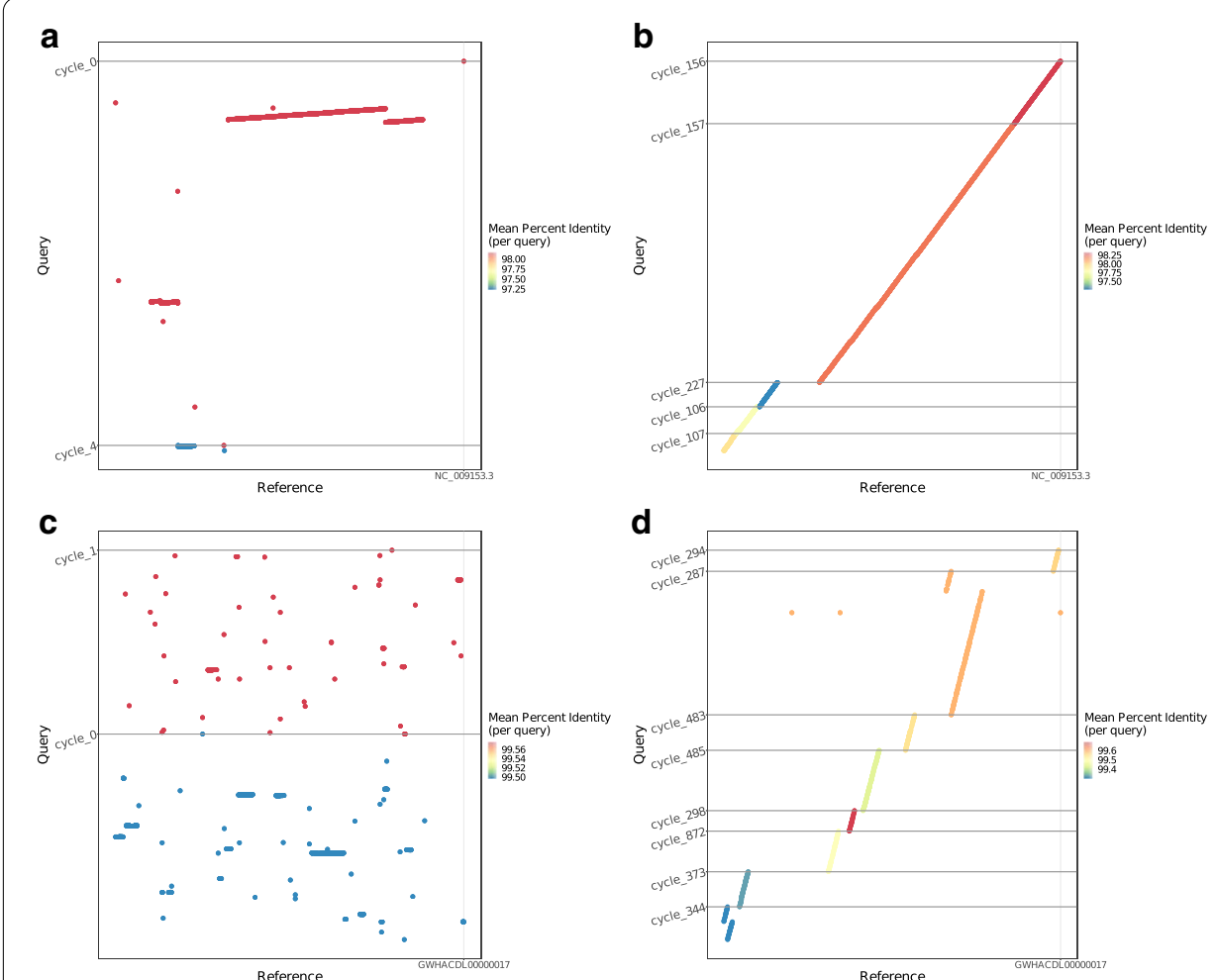

Fig. 3 Dot-plots showing alignments of re-assembled scaffolds by LDscaff versus chromosome-length scaffolds. Cutoff of 0.1 was used in $\mathbf{a}$ and $\mathbf{c}$, cutoff of 0.2 was used in $\mathbf{b}$ and $\mathbf{d}$. The panda reference chr 17 and the horse reference chr10 are shown on the $X$ axis in upper part and lower part, respectively. The $Y$ axis shows the re-assembled scaffolds (alignment longer than 5000bp, percentage of match higher than 70\%). They have been ordered and oriented to match the chromosomes as defined in the reference in order to facilitate comparison. Each dot represents the position of an individual resolved scaffold aligned to the reference. The color of the dots and lines were colored to represent the match rate for each scaffold (red indicate higher mat-ching rate, whereas blue indicates lower matching rate)
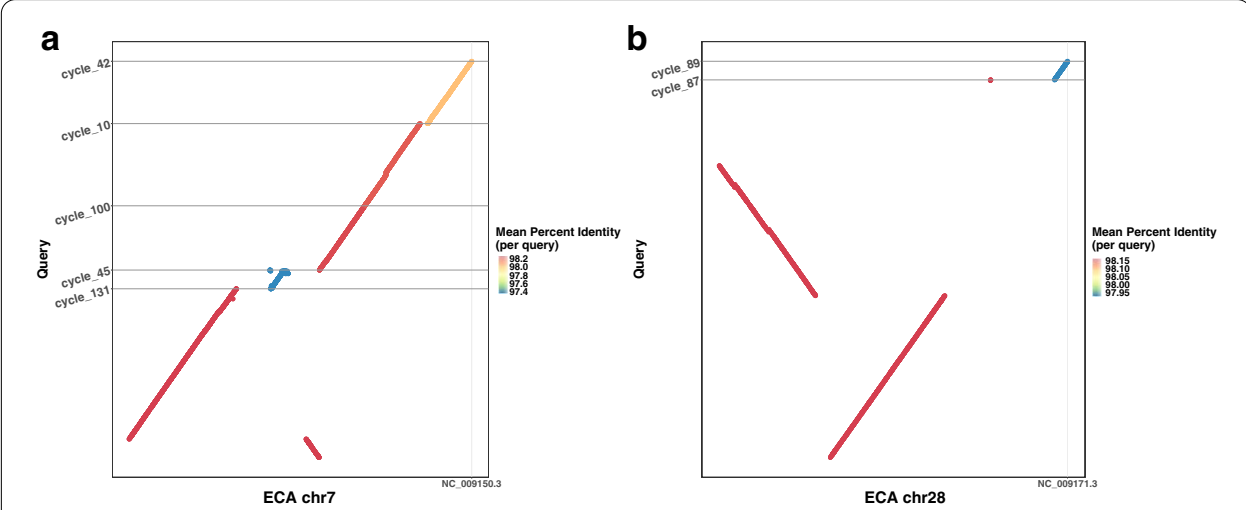

Fig. 4 Chromosomal inversions were identified using LDscaff a A inversion mapped to horse reference chr7; b A inversion mapped to horse reference chr28

integrated $\mathrm{HiC}$ and LDscaff, the assembly size has decreased to $1.8 \mathrm{G}$ from $2.2 \mathrm{G}$, while the N50 size has increased to $1.2 \mathrm{G}$, which is the size of the longest scaffold. 
Table 3 Mis-assembly comparison of the refined panda genome with SOAPdenovoassembled genome

\begin{tabular}{lll}
\hline Assembly evaluation & Before LDscaff & After LDscaff \\
\hline Misassemblies & 1092 & 448 \\
Contig misassemblies & 866 & 314 \\
C. relocations & 822 & 283 \\
c. translocations & 38 & 28 \\
$\quad$ c. inversions & 6 & 3 \\
Scaffold misassemblies & 226 & 134 \\
s. relocations & 220 & 126 \\
s. translocations & 6 & 8 \\
s. inversions & 0 & 0 \\
Misassembled contigs & 200 & 37 \\
Misassembled contigs length & 211889858 & 104831721 \\
Local misassemblies & 8122 & 2873 \\
Scaffold gap ext. mis. & 71 & 21 \\
Scaffold gap loc. mis. & 4856 & 1792 \\
Unaligned mis. contigs & 1502 & 563 \\
Mismatches & 412290 & 191921 \\
Indels & 95351 & 46834 \\
Indels ( $\leq 5$ bp) & 67654 & 33342 \\
Indels (> 5 bp) & 27697 & 13492 \\
Indels length & 896205 & 436349 \\
\hline
\end{tabular}

Table 4 Comparison of the refined donkey genome with SOAPdenovo-assembled genome

\begin{tabular}{lllll}
\hline Cutoff & Scaffolds before & Scaffolds after & N50 before & N50 after \\
\hline 0.1 & 80 & 81 & 23779253 & 2108947689 \\
0.15 & 80 & 254 & 23779253 & 44040746 \\
0.2 & 80 & 382 & 23779253 & 32092974 \\
0.3 & 80 & 484 & 23779253 & 26289196 \\
0.4 & 80 & 514 & 23779253 & 24644580 \\
0.5 & 80 & 542 & 23779253 & 23779253 \\
\hline
\end{tabular}

\section{Discussion}

Assemble the sequenced reads to chromosome level is a long term puzzle in genome analysis. The Human Genome Project tool scientists about 10 years to complete that first human genome sequence. Currently, tools are capable of obtaining a more accurate human genome with only hundreds of CPU hours [46].

With the explosive volume of sequencing data, it is important to take full advantage of the features in different sequencing strategies to achieve the task of genome assembly. Short reads provide accurate base calls while long reads can help to reconstruct the long-range structure of the genome [47]. Linked-Reads group reads deriving from the same molecule [48]. Hi-C data can provide linkage information across a variety of length scales. LD from population SNP data offers patterns of recombination rates. Hybrid assembly tools have been developed for integrating all these sequencing techniques [49]. Most of them are based on a clustering-assembly strategy by solving the 
Table 5 Mis-assembly comparison of the refined donkey genome with SOAPdenovoassembled genome

\begin{tabular}{lll}
\hline Assembly evaluation & Before LDscaff & After LDscaff \\
\hline Misassemblies & 7166 & 3266 \\
Contig misassemblies & 5173 & 2330 \\
C. relocations & 1517 & 651 \\
C. translocations & 3319 & 1522 \\
$\quad$ c. inversions & 337 & 157 \\
Scaffold misassemblies & 1993 & 936 \\
s. relocations & 425 & 215 \\
s. translocations & 1543 & 712 \\
s. inversions & 25 & 9 \\
Misassembled contigs & 369 & 122 \\
Misassembled contigs length & 2256451641 & 1112560100 \\
Local misassemblies & 59490 & 26941 \\
Scaffold gap ext. mis. & 80 & 31 \\
Scaffold gap loc. mis. & 26321 & 12206 \\
Unaligned mis. contigs & 18 & 26 \\
Mismatches & 27642582 & 13299554 \\
Indels & 2673003 & 1283993 \\
Indels (<5 bp) & 2189565 & 1051168 \\
Indels (> 5 bp) & 483438 & 232825 \\
Indels length & 13686335 & 6581365 \\
\hline
\end{tabular}

scaffold orientation and order asynchronously. It has been approved that the hybrid

Table 6 Assembly comparison after spliting by continuous fragments

\begin{tabular}{llll}
\hline Assembly composition & SOAPdenovo + LDscaff & SOAPdenovo + HiC & SOAPdenovo + LDscaff + HiC \\
\hline Assembly size & 2266537396 & 2252886107 & 1797011536 \\
No. scaffolds & 382 & 647 & 123 \\
N50 size & 32092974 & 58665385 & 1254064180 \\
Misassemblies & 2426 & 4852 & 2162 \\
Contig misassemblies & 2426 & 4852 & 2162 \\
$\quad$ c. relocations & 589 & 1231 & 524 \\
$\quad$ c. translocations & 1678 & 3298 & 1490 \\
$\quad$ c. inversions & 159 & 323 & 148 \\
Scaffold misassemblies & 0 & 0 & 0 \\
$\quad$ s. relocations & 0 & 0 & 0 \\
$\quad$ s. translocations & 0 & 0 & 0 \\
$\quad$ s. inversions & 0 & 0 & 0 \\
Misassembled contigs & 1528 & 3120 & 1384 \\
Misassembled contigs length & 71602181 & 144952053 & 67084793 \\
Local misassemblies & 28366 & 58360 & 27273 \\
Unaligned mis. contigs & 117 & 203 & 102 \\
Mismatches & 13257465 & 27321894 & 12732835 \\
Indels & 1249703 & 2585745 & 1204396 \\
Indels (<5 bp) & 1041691 & 2156454 & 1004988 \\
Indels (> 5 bp) & 208012 & 429291 & 199408 \\
Indels length & 5760596 & 11919093 & 5519229 \\
\hline
\end{tabular}


strategy can boost the assembly result in both continuity and accuracy.

LD indicates the non-random associations between physical markers. Linkage information among genes or loci provides relative position information [23] and is capable of finding scaffolds that conflict with their relative orders. Genetic linkage maps have been used to refine the de novo genome assemblies $[6,50]$, and show the potential to guide the layout of scaffolds. However, with millions of genomes are now being collected, the information provided by population data has not been fully utilized to resolve the task of genome assembly. Here we proposed a computational graph-based algorithm to resolve the scaffold orders and orientation simultaneously. Our method proves to be effective when applied to both simulated data and empirical data.

The sample size affects LD estimation. Theoretically, more individuals sampled leads to better performance. Two main methods are used to calculate LD, $r^{2}$ and $\left|D^{\prime}\right| . r^{2}$ has been proved not noticeably affected by sample size [51], which LDscaff uses. A minimum sample size of 55 for accurate calculation of LD is suggested [51].

Linkage information can help increase the continuity of assembly, but locus pairs with too long distances between them provide weak linkage power. Thereby we cut the unreliable links with a proper cutoff threshold. We advance the cutoff threshold should be equal to or larger than 0.2. The larger the cutoff threshold, the less the assembled scaffold contiguity, but better accuracy.

\section{Conclusions}

We hope that the improvement in genome assembly provided by LDscaff will further boost the use of existed sequencing data. While assemblers improve genome assemblies, data they require is the cost to be considered. Therefore, LDscaff provides an example to improve assembly quality by mining biological databases.

\section{Supplementary information}

is available for this paper at https://doi.org/10.1186/s12859-020-03895-7.

Additional file 1. The dot plots of LD-scaff re-assemblied scaffolds in Panda and Donkey genome.

\section{Abbreviations}

NGS: Next Generation Sequencing; LD: Linkage disequilibrium; WGS: Whole genome sequencing; SRA: Short Read Archive; PigVar: Pig variations and Positive Selection.

\section{Acknowledgements}

We thanked Yibo Hu (Institute of Zoology, Chinese Academy of Sciences) for sharing the panda GWHACDL00000000 assembly.

About this supplement

This article has been published as part of BMC Bioinformatics Volume 21 Supplement 21 2020: Accelerating Bioinformatics Research with ICIBM 2020. The full contents of the supplement are available at https://bmcbioinformatics.biome dcentral.com/articles/supplements/volume-21-supplement-21.

\section{Authors' contributions}

SL designed the research. ZZ and YZ performed the experiments and wrote the manuscript. SL, CW, SW and XZ reviewed the manuscript. All authors read and approved the final manuscript.

\section{Funding}

This work and its publication costs were funded by the National Natural Science Foundation of China (Grant No. 31671287 and 31601007), Well-bred Program of Shandong Province (Grant No. 2017LZGC020), Taishan Leading Industry Talents- Agricultural Science of Shandong Province (Grant No. LJNY201713), and Shandong Province Modern Agricultural 
Technology System Donkey Industrial Innovation Team (Grant No. SDAIT-27). The funding bodies did not play any role in the design of this study, nor the collection, analysis, or interpretation of data, nor in writing this manuscript.

\section{Availability of data and materials}

The swine WGS data were collected from the Pig variations and Positive Selection (PigVar) database, the swine assembly (Sus scrofa 10.2) can be obtained under GCA_000003025.4. We downloaded the giant panda reference (AilMel 1.0) from the NCBI GenBank database (Accession number: GCA000004335.1). We downloaded the panda GWHACDL00000000 assembly from National Genomics Data Center. The panda WGS data can be obtained under accession SRA053353. The data sets of donkey individuals generated and analyzed during the current study are not publicly available due to these data have been cited from another paper that is being peer-reviewed. Data sharing will be applied later. Project name: LDscaff; Project home page: https://github.com/YingXiaoZhou/LDscaff; Operating system(s): Platform independent; Programming language: Shell script, $c++$; Other requirements: lemon library installed; License: see web page; Any restrictions to use by non-academics: licence needed.

\section{Ethics approval and consent to participate}

Animal care and research procedures were carried out in accordance with the guiding principles for the care and use of laboratory animals, being approved by the Institutional Animal Care and Use Committee at Shandong Academy of Agricultural Sciences (SAAS) for the donkey individuals involved in this study.

\section{Consent to publish}

Not applicable.

\section{Competing interests}

The authors declare that they have no competing interests.

\section{Author details}

${ }^{1}$ BGI Education Center, University of Chinese Academy of Sciences, Shenzhen 518083, China. ${ }^{2}$ Department of Computer Science, City University of Hong Kong, Kowloon, Hong Kong SAR 999077, China. ${ }^{3}$ Liaocheng Research Institute of Donkey High-Efficiency Breeding and Ecological Feeding, Liaocheng University, Liaocheng City 252059, Shandong, China. ${ }^{4}$ BGI-Shenzhen, Shenzhen 518083, China.

Received: 17 November 2020 Accepted: 18 November 2020

Published: 28 December 2020

\section{References}

1. Gnerre S, MacCallum I, Przybylski D, Ribeiro FJ, Burton JN, Walker BJ, Sharpe T, Hall G, Shea TP, Sykes S, et al. Highquality draft assemblies of mammalian genomes from massively parallel sequence data. Proc Natl Acad Sci. 2011;108(4):1513-8.

2. Jex AR, Liu S, Li B, Young ND, Hall RS, Li Y, Yang L, Zeng N, Xu X, Xiong Z, et al. Ascaris suum draft genome. Nature. 2011;479(7374):529.

3. Kim EB, Fang X, Fushan AA, Huang Z, Lobanov AV, Han L, Marino SM, Sun X, Turanov AA, Yang P, et al. Genome sequencing reveals insights into physiology and longevity of the naked mole rat. Nature. 2011;479(7372):223.

4. Li R, Fan W, Tian G, Zhu H, He L, Cai J, Huang Q, Cai Q, Li B, Bai Y, et al. The sequence and de novo assembly of the giant panda genome. Nature. 2010;463(7279):311.

5. Murchison EP, Schulz-Trieglaff OB, Ning Z, Alexandrov LB, Bauer MJ, Fu B, Hims M, Ding Z, Ivakhno S, Stewart C, et al. Genome sequencing and analysis of the tasmanian devil and its transmissible cancer. Cell. 2012;148(4):780-91.

6. Potato Genome Sequencing Consortium. Genome sequence and analysis of the tuber crop potato. Nature. 2011:475(7355):189.

7. Vinson JP, Jaffe DB, O'Neill K, Karlsson EK, Stange-Thomann N, Anderson S, Mesirov JP, Satoh N, Satou Y, Nusbaum C, et al. Assembly of polymorphic genomes: algorithms and application to Ciona savignyi. Genome Res. 2005;15(8):1127-35.

8. Al-Dous EK, George B, Al-Mahmoud ME, Al-Jaber MY, Wang H, Salameh YM, Al-Azwani EK, Chaluvadi S, Pontaroli AC, DeBarry J, et al. De novo genome sequencing and comparative genomics of date palm (Phoenix dactylifera). Nat Biotechnol. 2011;29(6):521.

9. Sohn J-I, Nam J-W. The present and future of de novo whole-genome assembly. Brief Bioinform. 2016;19(1):23-40.

10. Li R, Zhu H, Ruan J, Qian W, Fang X, Shi Z, Li Y, Li S, Shan G, Kristiansen K, et al. De novo assembly of human genomes with massively parallel short read sequencing. Genome Res. 2010;20(2):265-72.

11. Alkan C, Sajjadian S, Eichler EE. Limitations of next-generation genome sequence assembly. Nat Methods. 2011;8(1):61.

12. Baker SC. Next-generation sequencing challenges. Genet Eng Biotechnol News. 2017;37(3):1-14.

13. Kitzman JO, Mackenzie AP, Adey A, Hiatt JB, Patwardhan RP, Sudmant PH, Ng SB, Alkan C, Qiu R, Eichler EE, et al. Haplotype-resolved genome sequencing of a Gujarati Indian individual. Nat Biotechnol. 2011;29(1):59.

14. Zhang G, Fang X, Guo X, Li L, Luo R, Xu F, Yang P, Zhang L, Wang X, Qi H, et al. The oyster genome reveals stress adaptation and complexity of shell formation. Nature. 2012;490(7418):49.

15. Lam ET, Hastie A, Lin C, Ehrlich D, Das SK, Austin MD, Deshpande P, Cao H, Nagarajan N, Xiao M, et al. Genome mapping on nanochannel arrays for structural variation analysis and sequence assembly. Nat Biotechnol. 2012;30(8):771.

16. Dong Y, Xie M, Jiang Y, Xiao N, Du X, Zhang W, Tosser-Klopp G, Wang J, Yang S, Liang J, et al. Sequencing and automated whole-genome optical mapping of the genome of a domestic goat (Capra hircus). Nat Biotechnol. 2013;31(2):135. 
17. Zhang $Q$, Chen W, Sun L, Zhao F, Huang B, Yang W, Tao Y, Wang J, Yuan Z, Fan G, et al. The genome of prunus mume. Nat Commun. 2012;3:1318.

18. Baird NA, Etter PD, Atwood TS, Currey MC, Shiver AL, Lewis ZA, Selker EU, Cresko WA, Johnson EA. Rapid SNP discovery and genetic mapping using sequenced RAD markers. PLoS ONE. 2008;3(10):3376.

19. Burton JN, Adey A, Patwardhan RP, Qiu R, Kitzman JO, Shendure J. Chromosome-scale scaffolding of de novo genome assemblies based on chromatin interactions. Nat Biotechnol. 2013;31(12):1119.

20. Slatkin M. Linkage disequilibrium-understanding the evolutionary past and mapping the medical future. Nat Rev Genet. 2008;9(6):477.

21. Abecasis GR, Cookson W. Gold-graphical overview of linkage disequilibrium. Bioinformatics. 2000;16(2):182-3.

22. Jorde L, Watkins W, Carlson M, Groden J, Albertsen H, Thliveris A, Leppert M. Linkage disequilibrium predicts physical distance in the adenomatous polyposis coli region. Am J Hum Genet. 1994;54(5):884.

23. Khatkar MS, Hobbs M, Neuditschko M, Sölkner J, Nicholas FW, Raadsma HW. Assignment of chromosomal locations for unassigned SNPs/scaffolds based on pair-wise linkage disequilibrium estimates. BMC Bioinform. 2010;11(1):171.

24. Pengelly RJ, Collins A. Linkage disequilibrium maps to guide contig ordering for genome assembly. Bioinformatics. 2019;35(4):541-5.

25. Mascher M, Muehlbauer GJ, Rokhsar DS, Chapman J, Schmutz J, Barry K, Muñoz-Amatriaín M, Close TJ, Wise RP, Schulman AH, et al. Anchoring and ordering NGS contig assemblies by population sequencing (POPSEQ). Plant J. 2013;76(4):718-27.

26. Hahn MW, Zhang SV, Moyle LC. Sequencing, assembling, and correcting draft genomes using recombinant populations. G3 Genes Genomes Genet. 2014;4(4):669-79.

27. Nossa CW, Havlak P, Yue J-X, Lv J, Vincent KY, Brockmann HJ, Putnam NH. Joint assembly and genetic mapping of the Atlantic horseshoe crab genome reveals ancient whole genome duplication. GigaScience. 2014;3(1):2047-217.

28. Schook LB, Beever JE, Rogers J, Humphray S, Archibald A, Chardon P, Milan D, Rohrer G, Eversole K. Swine genome sequencing consortium (SGSC): a strategic roadmap for sequencing the pig genome. Int J Genomics. 2005;6(4):251-5.

29. Fan H, Wu Q, Wei F, Yang F, Ng BL, Hu Y. Chromosome-level genome assembly for giant panda provides novel insights into carnivora chromosome evolution. Genome Biol. 2019;20(1):1-12.

30. Luo R, Liu B, Xie Y, Li Z, Huang W, Yuan J, He G, Chen Y, Pan Q, Liu Y, et al. SOAPdenovo2: an empirically improved memory-efficient short-read de novo assembler. Gigascience. 2012;1(1):18.

31. Li H, Durbin R. Fast and accurate short read alignment with burrows-wheeler transform. Bioinformatics. 2009;25(14):1754-60.

32. Li H, Handsaker B, Wysoker A, Fennell T, Ruan J, Homer N, Marth G, Abecasis G, Durbin R. The sequence alignment/ map format and SAMtools. Bioinformatics. 2009;25(16):2078-9.

33. Garrison E, Marth G. Haplotype-based variant detection from short-read sequencing. arXiv preprint arXiv:1207.3907 (2012).

34. Van der Auwera GA, Carneiro MO, Hartl C, Poplin R, Del Angel G, Levy-Moonshine A, Jordan T, Shakir K, Roazen D, Thibault J, et al. From FastQ data to high-confidence variant calls: the genome analysis toolkit best practices pipeline. Curr Protoc Bioinform. 2013;43(1):10-1.

35. Bolger AM, Lohse M, Usadel B. Trimmomatic: a flexible trimmer for illumina sequence data. Bioinformatics. 2014;30(15):2114-20.

36. Galil Z. Efficient algorithms for finding maximal matching in graphs. In: Colloquium on trees in algebra and programming. Springer; 1983. p. 90-113.

37. Madry A. Navigating central path with electrical flows: from flows to matchings, and back. In: 2013 IEEE 54th annual symposium on foundations of computer science (FOCS). IEEE; 2013. p. 253-62.

38. Mucha M, Sankowski P. Maximum matchings via Gaussian elimination. In: Proceedings of 45th annual IEEE symposium on foundations of computer science. IEEE; 2004. p. 248-55.

39. Dezső B, Jüttner A, Kovács P. LEMON-an open source C++ graph template library. Electron Notes Theor Comput Sci. 2011;264(5):23-45.

40. Marçais G, Delcher AL, Phillippy AM, Coston R, Salzberg SL, Zimin A. MUMmer4: a fast and versatile genome alignment system. PLoS Comput Biol. 2018;14(1):1005944.

41. Gurevich A, Saveliev V, Vyahhi N, Tesler G. QUAST: quality assessment tool for genome assemblies. Bioinformatics. 2013;29(8):1072-5.

42. Kurtz S, Phillippy A, Delcher AL, Smoot M, Shumway M, Antonescu C, Salzberg SL. Versatile and open software for comparing large genomes. Genome Biol. 2004;5(2):12.

43. Renaud G, Petersen B, Seguin-Orlando A, Bertelsen MF, Waller A, Newton R, Paillot R, Bryant N, Vaudin M, Librado P, et al. Improved de novo genomic assembly for the domestic donkey. Sci Adv. 2018;4(4):0392.

44. Dudchenko O, Batra SS, Omer AD, Nyquist SK, Hoeger M, Durand NC, Shamim MS, Machol I, Lander ES, Aiden AP, et al. De novo assembly of the Aedes aegypti genome using $\mathrm{Hi}-\mathrm{C}$ yields chromosome-length scaffolds. Science. 2017;356(6333):92-5.

45. Durand NC, Shamim MS, Machol I, Rao SS, Huntley MH, Lander ES, Aiden EL. Juicer provides a one-click system for analyzing loop-resolution Hi-C experiments. Cell Syst. 2016;3(1):95-8.

46. Ruan J, Li H. Fast and accurate long-read assembly with wtdbg2. Nat Methods. 2020;17(2):155-8.

47. Koren S, Schatz MC, Walenz BP, Martin J, Howard JT, Ganapathy G, Wang Z, Rasko DA, McCombie WR, Jarvis ED, et al. Hybrid error correction and de novo assembly of single-molecule sequencing reads. Nat Biotechnol. 2012;30(7):693.

48. Mostovoy Y, Levy-Sakin M, Lam J, Lam ET, Hastie AR, Marks P, Lee J, Chu C, Lin C, Džakula Ž, et al. A hybrid approach for de novo human genome sequence assembly and phasing. Nat Methods. 2016;13(7):587-90.

49. Antipov D, Korobeynikov A, McLean JS, Pevzner PA. HYBRIDSPADES: an algorithm for hybrid assembly of short and long reads. Bioinformatics. 2016;32(7):1009-15.

50. Kawakami T, Smeds L, Backström N, Husby A, Qvarnström A, Mugal CF, Olason P, Ellegren H. A high-density linkage map enables a second-generation collared flycatcher genome assembly and reveals the patterns of avian recombination rate variation and chromosomal evolution. Mol Ecol. 2014;23(16):4035-58. 
51. Bohmanova J, Sargolzaei M, Schenkel FS. Characteristics of linkage disequilibrium in North American Holsteins. BMC Genom. 2010;11(1):421.

\section{Publisher's Note}

Springer Nature remains neutral with regard to jurisdictional claims in published maps and institutional affiliations.

- fast, convenient online submission

- thorough peer review by experienced researchers in your field

- rapid publication on acceptance

- support for research data, including large and complex data types

- gold Open Access which fosters wider collaboration and increased citations

- maximum visibility for your research: over $100 \mathrm{M}$ website views per year

At BMC, research is always in progress.

Learn more biomedcentral.com/submissions 